\title{
The use of phage display to distinguish insulin autoantibody (IAA) from insulin antibody (IA) idiotypes
}

\author{
D. Devendra' 1 , T. S. Galloway ${ }^{3}$, S. J. Horton 1 , A. Evenden ${ }^{3}$, U. Keller ${ }^{4}$, T. J. Wilkin ${ }^{1}$ \\ ${ }^{1}$ Department of University Medicine, Plymouth Campus, United Kingdom \\ ${ }^{3}$ Department of Biological Sciences, University of Plymouth, Plymouth, United Kingdom \\ ${ }^{4}$ Division of Endocrinology and Metabolism, University of Basel, Basel, Switzerland
}

\section{Abstract}

Aim/hypothesis. Radiobinding assays (RBA) are unable to differentiate insulin autoantibodies (IAA) from insulin antibodies (IA). We sought to establish whether random peptide phage display might generate reagents with which to distinguish IAA idiotopes from IA idiotopes.

Methods. Two insulin-binding sera were used to select phagotopes from a phage library. The first, designated IAS, came from an insulin-treated patient with the insulin autoimmune syndrome, and was known to contain both IA and a high titre of human insulin specific (B30 threonine dependent) IAA. The second, designated IDD, was taken from a newly-diagnosed IAA+ Type 1 diabetic patient. Phage colonies selected by insulin-purified IgG extracts of IAS and IDD were selected at random for DNA sequencing, and tested for their reactivity with insulin antibodies and ability to distinguish disease-associated idiotopes.

Results. Seven phagotopes bound IAS and the phagotope designated IAS-9, corresponding to sequence
KRSRLDV, gave the highest binding standard deviation (SD) score. Seven phagotopes bound IDD and the phagotope designated IDD-10, corresponding to sequence LGRGGSK, bound most strongly. IAS-9 was able to displace insulin binding in IAS and all of ten insulin-treated Type 2 diabetic patients, but not the IAA present in any of the eight patients with newlydiagnosed Type 1 diabetes. IDD-10, on the other hand, could displace insulin binding detected in the sera of eight patients with untreated Type 1 diabetes (IAA), but not in IAS or sera of the insulin-treated Type 2 diabetics.

Conclusion. Phagotopes provide reagents which between them can distinguish positively as well as negatively diabetes-associated IAA from non-diabetes associated IAA and from IA. [Diabetologia (2003) 46:802-809]

Keywords Insulin autoantibody, insulin antibody, phage display, microfiltration radiobinding assay, type 1 diabetes, type 2 diabetes, insulin autoimmune syndrome, epitopes and idiotopes.
Received: 30 October 2002 / Revised: 17 February 2003

Published online: 3 June 2003

C) Springer-Verlag 2003

Corresponding author: Dr. D. Devendra, Barbara Davis Center for Childhood Diabetes, University of Colorado Health Science Center, 4200 East 9th Avenue, Box B-140, Denver, Colorado 80262, USA

E-mail: devasenan.devendra@uchsc.edu

Abbreviations: IA, insulin antibody; IAA, insulin autoantibody; IAS, insulin autoimmune syndrome; GAD, glutmate acid decarboxylase; IA-2, tyrosine like phosphatase; ICA, islet cell antibody; HLA, human leukocyte antigen.
Type 1 diabetes mellitus results from autoimmune destruction of the insulin-producing beta cells in the islets of Langerhans [1]. Autoimmune insulitis is accompanied from an early stage by the presence of circulating islet-related autoantibodies [2]. Several have been identified, but only insulin autoantibodies (IAA) bind an antigen specific to the beta cell.

IAA arise, by definition, in individuals who have never been exposed to exogenous insulin [3], in contrast to insulin antibodies (IA) which are induced through the administration of therapeutic insulin. IAA is of particular interest as markers for beta-cell disease, and is the first of the islet-related antibodies to 
appear during early insulitis [4]. Insulin binding antibodies can nevertheless be isolated from healthy people, from patients with autoimmune diseases other than Type 1 diabetes [5], and following certain medications where their presence reduces the clinical specificity of the test for diabetes.

In their present form, radiobinding assays (RBA) [6, $7,8]$ do not distinguish the epitope of the insulin molecule with which the antibody binds, and cannot define the antibody idiotype involved in the reaction. There is serological evidence that IAA idiotypes could be disease-specific $[9,10]$ and assay technology able to discriminate the disease-predictive idiotypes of IAA from non disease-predictive IAA and from IA would be an important advance for the specificity of the test.

The insulin molecule carries around 120 different potential epitopes to which antibodies can be produced during the immune response [11]. Studies in which the epitope recognition by IA and IAA have been compared in the context of diabetes have shown a highly variable response in which hydrophilic surface residues of both $\mathrm{A}$ and $\mathrm{B}$ chains are frequently targeted $[12,13]$. The insulin autoimmune syndrome (IAS) is characterized by IAA-mediated disturbances of insulin kinetics in patients who have not previously received exogenous insulin [14], and a characteristic feature in some patients is the appearance of high affinity monotypic responses restricted to particular epitopes on the insulin molecule.

We have previously investigated the serum from one such patient with IAS whose IAA were human insulin specific [15]. Human differs from porcine insulin by the single substitution of threonine for alanine at position $\mathrm{B} 30$, so that two naturally occurring insulins are all that is needed to positively distinguish these particular IAA from IA, though not vice versa given that human insulin is bound by both. Such sera offer an ideal opportunity to examine epitope/idiotope responses in the context of autoimmunity, but conventional serology alone is limited as a technology.

An alternative method for defining the nature of antibody:antigen interactions is to probe phage-display peptide libraries with the antibody in question. The technique can show both conformational and linear phagotopes that mimic the shape of the conformational epitopes [16]. Conformational epitopes have been identified by this method using monoclonal and polyclonal antibodies in both animal and human sera $[17,18]$. The term phagotope is used in preference to mimotope here, as the binding properties of the displayed peptides are usually studied in the context of the entire phage particle [19]. The idiotype of an antibody is the sum of the antibody variable region determinants used to bind antigen. The idiotype, and the epitope of the ligand to which it binds, can be defined only at the molecular level and this paper reports our attempts to distinguish the epitopes recognised by insulin antibodies from different disease states.

\section{Subjects and methods}

We set out to establish proof of principle that phage display technology can create and sequence a library of phagotopes able to distinguish the idiotypes of diabetes-associated IAA from those of other IAA and IA. To do so, we used the serum from a patient with IAS and from another at onset of Type 1 diabetes to select candidate phagotopes which we went on to test clinically. The phagotopes were amplified, checked for their ability to bind specifically the sera used to select them, investigated for their wider ability to displace insulin binding in antibody-containing sera from people with Type 1 and insulin-treated Type 2 diabetes, and sequenced.

Sera used to select phagotopes. Two high-titre sera were used, shown to bind human insulin in a microfiltration RBA. The first, designated IAS, was the serum we have described previously, chosen because of the mixture it contained of spontaneously occurring human insulin-specific IAA and IA in response to therapeutic insulin. One of the challenges was to develop regents able to distinguish one from the other. The serum was taken from a 69-year-old woman with the insulin autoimmune syndrome who had begun insulin treatment on account of gradually rising blood sugars following partial pancreatectomy. Her IAA were displaceable in an RBA by human insulin, but only partially by bovine or porcine insulin. Her HLA status was $A * 30 A * 32 B 27 B 62(15) D R B 1 * 01 D R B 1 * 16$. The second serum, designated IDD, was collected from a five-year old girl at onset of Type 1 diabetes, prior to her first injection of insulin. She was IAA, GAD and IA-2 antibody positive. Her genotype was $D R * 0401-D Q A 1 * 05-D Q B 1 * 0301 / * 0302$.

Sera used to test the binding of phagotopes selected by IAS and IDD were obtained from (i). Ten normal subjects (mean age 49.5 SD \pm 6.5 years) who were selected as controls. They had not been exposed to insulin, and had no personal or family history of diabetes or of other endocrine disorders. (ii) Eight children with newly diagnosed Type 1 diabetes, prior to insulin injection, (mean age 10.3 SD \pm 2.9 years). (iii) Ten adults with Type 2 diabetes (mean age $64.6 \mathrm{SD} \pm 11.3$ years) who had been treated with insulin for than 6 months or more. The GAD antibody status was negative in all ten patients but ICA and IA-2 were not tested in these individuals.

The local ethics committee approved the study and all subjects (or parents as appropriate) gave informed consent.

Microfiltration Radiobinding Assay for IA and IAA. We used a modification of a known method for the detection of insulin binding in IAS, IDD and sera from the 10 controls, the eight Type 1 and the 10 insulin-treated patients [8]. Briefly, serum $(5 \mu \mathrm{l})$ was incubated for $72 \mathrm{~h}$ at $4{ }^{\circ} \mathrm{C}$ in duplicate wells in a prewetted millipore filtration microplate $(0.45 \mu \mathrm{m})$ with $15,000 \mathrm{cpm}$ A-14 monoiodinated recombinant human insulin (Amersham Biosciences, Bucks,U.K) $(1.2 \mathrm{MBq})$ in $25 \mu \mathrm{l}$ $50 \mathrm{mmol} / \mathrm{l}$ Tris pH 8.0 containing $1 \%$ v/v Tween-20 (TBT). Phase separation was achieved using $8 \%$ Protein G Sepaharose in $50 \mu \mathrm{l} \mathrm{TBT}$. After $90 \mathrm{~min}$, wells were aspirated by vacuum filtration and washed five times with $200 \mu$ ice cold TBT. Optiphase Supermix (Wallac, Turku, Finland) scintillation fluid $(50 \mu \mathrm{l})$ was added and the plates counted in a microplate counter (Wallac Microbeta,Turku, Finland). The binding of serum to radiolabelled insulin was expressed as a percentage of binding (cpm of radiolabel insulin after protein $\mathrm{A} / \mathrm{G}$ precipitation in test well-mean cpm of ten controls / 15,000 cpm). To test the extent to which the introduction of therapeutic insulin to treat her diabetes had altered the previously exclusively hu- 
man insulin specificity of IAS, we attempted to displace the binding with human, porcine and bovine insulin at various molarities. The molarity of insulin needed to displace insulin binding by $50 \%\left(\mathrm{D}_{50}\right)$ was used to compare the avidities of the serum for the three insulin variants. The inter-assay and intraassay co-efficient variation of the method was $9.3 \%$ and $9.5 \%$ respectively.

Affinity purification of IAS and IDD for phage library screening. Prior to using IAS and IDD for phage selection, the sera were subjected to a process of purification. Immunoglobulins were first separated by precipitation with ammonium sulphate at a final concentration of $50 \%(\mathrm{w} / \mathrm{v})$. An initial volume of $0.4 \mathrm{ml}$ of sera was used from each subject. Precipitated protein was pelleted by centrifugation $(5000 \times \mathrm{g}, 10 \mathrm{~min})$ and washed twice with $50 \%$ ammonium sulphate. The pellet was re-suspended in phosphate buffer saline $\mathrm{pH} 7.4$, and dialysed overnight.

The partially purified immunoglobulin fraction was further purified to extract IgG by affinity chromatography using Protein G (HiTrap Affinity Protein G, Amersham Biosciences) following the manufacturer's instructions. The column $(1 \mathrm{ml})$ was equilibrated with three volumes of start buffer containing $20 \mathrm{mmol} / \mathrm{l}$ sodium phosphate $\mathrm{pH}$ 7.0. The sample was applied, washed with a further five volumes of wash buffer and the bound immunoglobulin eluted with three volumes of $0.1 \mathrm{mmol} / \mathrm{l}$ glycine_HCl, $\mathrm{pH} 2.7$. Fractions of $0.5 \mathrm{ml}$ were collected into $0.1 \mathrm{ml} 1 \mathrm{~mol} / \mathrm{l}$ Tris, $\mathrm{pH} 8.0$ and were tested for total protein (BioRad, Herts, UK) using bovine serum albumin as standard and for insulin binding by RBA as described. This aliquot was further enriched for insulin antibodies by passing it through an affinity column containing cyanogen bromide activated matrices covalently linked to human recombinant insulin (Sigma-Aldrich, Gillingham, UK). In preparing the column, insulin was coupled to the cyanogen-bromide activated sepharose (4 Fast Flow, Sigma-Aldrich) using $0.1 \mathrm{mmol} / 1$ sodium bicarbonate buffer containing $0.5 \mathrm{mmol} / \mathrm{l}$ sodium chloride, $\mathrm{pH} 8.5$ to give $10 \mathrm{mg}$ of protein/ml of gel. After standing, unbound ligand was washed through and non-reactive groups blocked with $0.2 \mathrm{mmol} / \mathrm{l}$ glycine, $\mathrm{pH}$ 8.0. Following extensive washing with coupling buffer and $0.1 \mathrm{mmol} / \mathrm{l}$ acetate buffer, $\mathrm{pH} 4.0$ (containing $0.5 \mathrm{mmol} / \mathrm{l} \mathrm{NaCl}$ ) the column was finally equilibrated for immediate use with several volumes of start buffer, $20 \mathrm{mmol} / 1$ sodium phosphate at $\mathrm{pH}$ 7.0. The eluted fraction was tested for total protein and insulin binding. The purity and molecular weight of the immunoglobulin preparations was determined by $10 \%$ sodium dodecyl sulphate polyacrylamide gel electrophoresis (SDS-PAGE) using silver staining (SigmaAlrich). The total amount of protein recovered for IAS was $3.2 \mu \mathrm{g}$ and for IDD was $2.8 \mu \mathrm{g}$. The purified protein was retested for its insulin binding properties using the RBA described above.

Phage display random peptide library. The $\mathrm{Ph} \mathrm{D}-7$ random heptapeptide library was purchased from New England Biolabs (Beverly, Mass., USA). The library contained $2 \times 10^{13}$ plaqueforming units (PFU)/ml with a complexity of $2 \times 10^{9}$ combination of 7-mers. The displayed heptapeptides were expressed at the amino terminus of the pIII coat protein of the filamentous coliphage M13, and the library was used at a concentration of $8 \times 10^{10} \mathrm{PFU} / \mathrm{ml}$. The library was stored in Tris-buffered saline (TBS, $50 \mathrm{mmol} / \mathrm{l}$ Tris at $\mathrm{pH} 7.4$ in $0.9 \% \mathrm{NaCl}$ ) with $50 \%$ glycerol. Phage was propagated in E. coli strain ER2537, which was provided with the library kit as a non-competent glycerol stock. The DNA inserts from individual non-selected colonies were sequenced from the library to confirm that they contained random peptide sequences.
Biopanning and isolation of phage. The library was screened by biopanning, using the insulin purified $\operatorname{IgG}$ described above. The IgG $(300 \mathrm{ng})$ from the IAS patient was incubated overnight at $4{ }^{\circ} \mathrm{C}$ with $10 \mu \mathrm{l}$ of the phagemid library in $500 \mu \mathrm{l}$ of PBS, pH 7.3 containing $1 \mathrm{mg} / \mathrm{ml}$ bovine serum albumin (PBSBSA). Phages that bound the $\mathrm{IgG}$ were isolated using Protein G (Sigma-Aldrich) precipitation and washed ten times in PBSBSA. The phages were subsequently eluted from the protein $G$ with $1 \mathrm{mg} / \mathrm{ml} \mathrm{BSA}$ in $0.1 \mathrm{~mol} / 1 \mathrm{HCl}$ at $\mathrm{pH} 2.2$, adjusted with glycine, and then neutralized with $1 \mathrm{~mol} / \mathrm{l}$ Tris- $\mathrm{HCl}, \mathrm{pH} 9$. After this first round of "positive" selection, there followed a "negative" selection step in which phages not specifically reactive with IAA sera were removed using $100 \mu \mathrm{g}$ of IgG prepared from pooled normal sera.

Amplification of phage. The phages were amplified by infecting E. coli EWR2537 cells in the logarithmic phase of growth and allowing them to grow at $37^{\circ} \mathrm{C}$ with shaking for $4.5 \mathrm{~h}$. Phages from liquid culture were obtained by clearing the supernatant twice by centrifugation at $13,000 \times g$ for $5 \mathrm{~min}$. Phage particles were precipitated with $4 \%$ polyethylene glycol 6000 in $0.5 \mathrm{~mol} / 1 \mathrm{NaCl}$, centrifuged at $13,000 \times \mathrm{g}$ for $15 \mathrm{~min}$, and the pellets were re-suspended in $1 \mathrm{ml}$ TBS. Two further rounds of positive selection, negative selection, and amplification enriched the amplified eluate. Each biopanning involved three rounds of positive selection with IgG from IAS and IDD.

Immunospecificity of reaction between phage and IAS/IDD. Ten random phage recombinant clones selected from biopanning with IAS and and ten with IDD were tested for their capacity to bind IAS and IDD immunospecifically in a capture ELISA. The two sets of ten phagotopes were designated IAS 1-10 and IDD 1-10 respectively. The capture ELISA followed a protocol used by another study to detect phagotopes from phage display [20]. A 96-well microtitre plate was coated with $100 \mu \mathrm{l} /$ well of affinity-purified preparations of either IAS or IDD in sterile PBS, pH 7.3 and incubated overnight at $4^{\circ} \mathrm{C}$ in a humidified chamber. Sera (diluted 1:500) from ten IAA/IA negative patients were used as controls. The plates were blocked for $2 \mathrm{~h}$ at room temperature with $1 \%$ skimmed milk powder in $0.05 \%$ Tween 20 in PBS $(200 \mu \mathrm{l} /$ well $)$ and washed three times with $0.005 \%$ Tween 20 in TBS, pH 7.4. Duplicate samples $(100 \mu \mathrm{l})$ of purified phage diluted 1:20 in sterile PBS were added to the wells and incubated overnight at $4^{\circ} \mathrm{C}$. The plates were blocked and washed as before. IgG was detected using HRP/ mouse anti-M13 monoclonal conjugated in $0.03 \mathrm{~mol} / \mathrm{l}$ citric acid, $0.04 \mathrm{~mol} / 1 \mathrm{Na}_{2} \mathrm{HPO}_{4}$, and $0.003 \% \mathrm{H}_{2} \mathrm{O}_{2}$, $\mathrm{pH} 4$ as substrate. In order to account for non-specific binding, the difference in the absorbance signal between the wells containing purified IAS or IDD and the mean of the ten controls was expressed as a SD score. A signal greater than the mean +2 SD (2 SDS) was considered positive. The inter-assay and intra-assay co-efficient of variation of the method was $10.2 \%$ and $11.5 \%$ respectively.

Ability of phagotopes to discriminate between IA and IAA in patient sera. A protocol similar to the microfiltration RBA was used but, instead of using cold insulin as a ligand to displace insulin binding, the phagotopes with the highest and the lowest SD scores in the capture ELISA were tested. Accordingly, two IAS and two IDD phages were used in dilutions of 1:1, 1:10, $1: 100,1: 1000$ and $1: 10,000$ to displace insulin binding in eight patients with newly diagnosed Type 1 diabetes and in ten patients with Type 2 diabetes treated with insulin. The concentration of phage colonies was calculated at 1:1 dilution to ensure that the concentration was similar in each phagotope selected prior to displacement reactions. 
A
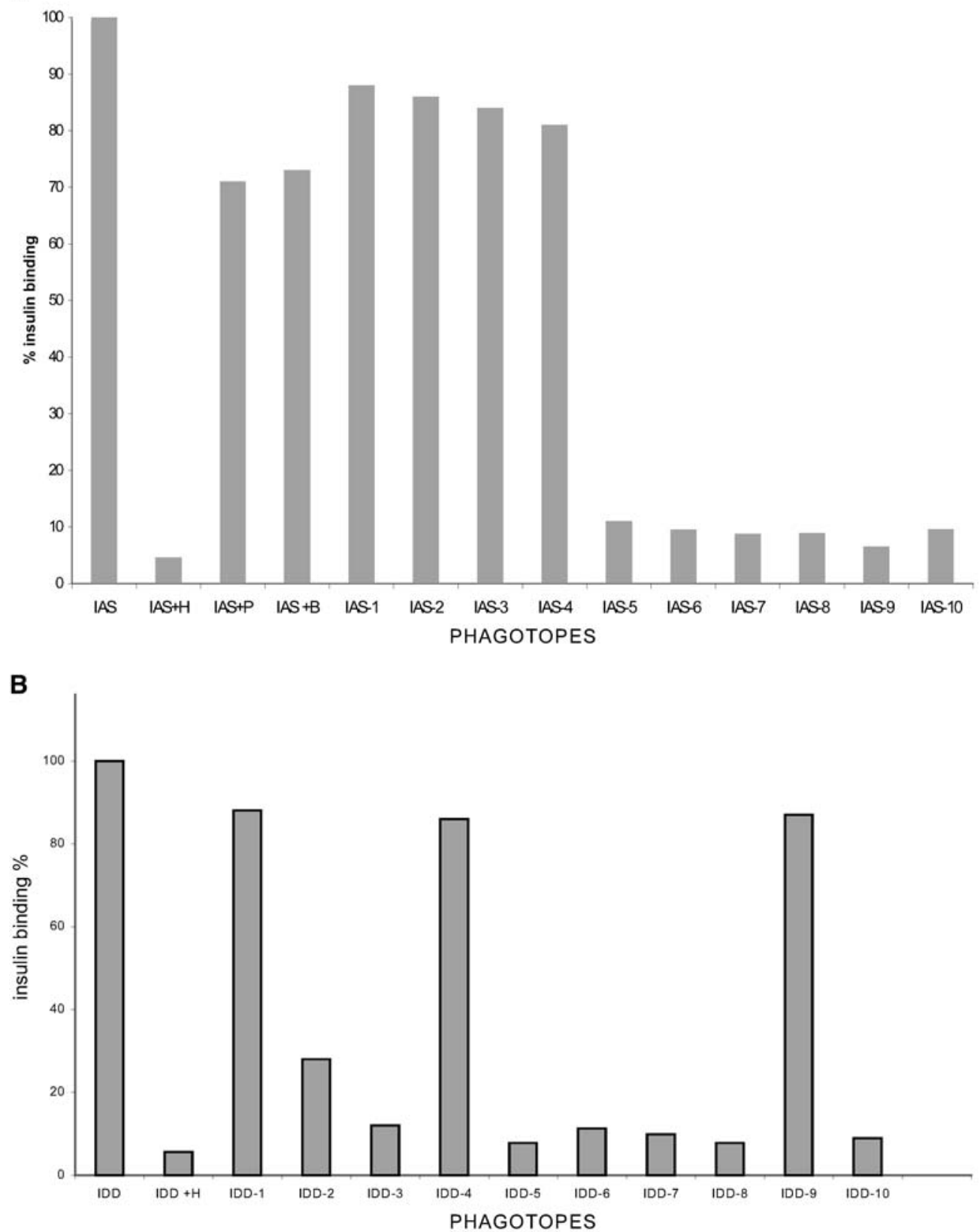

Fig. 1A, B. Comparison of the abilities of three insulin variants and selected phagotopes (dilution 1:100) to displace binding of labeled human insulin to serum IAS (A) and IDD (B). A * IAS: detection of insulin binding in the sera of the patient described with Insulin Autoimmune Syndrome. † IAS + H (human), P (porcine) or B (beef) insulin: Sera of the IAS patient incubated with different insulin species to displace insulin binding. $\div$ IAS-1 to 10 : phagotopes sequenced from the serum of the patient with IAS. B IDD: detection of insulin binding in the sera of the IDD patient. $\dagger$ IDD,$+ \mathrm{H}$ (human): Sera of the IDD patient incubated with human specific insulin to displace insulin binding. $\div$ IDD-1 to 10 : phagotopes sequenced from the serum of the IDD patient
DNA sequencing. IAS 1-10 and IDD 1-10 were sequenced. Single-stranded DNA was prepared using the QIA PREP spin M13 kit (Qiagen, Crawley, UK). The DNA templates was concentrated by ethanol extraction and sent to MWG Biotech (Dusseldorf, Germany) for sequencing. The non-standard primer used in this kit was $-965^{\prime}$ HO CCC TCA TAG TTA GCG TAA CG-3'. The peptide sequences and the consensus observed from the alignment of the phagotopes were mapped against the insulin $\mathrm{A}$ and $\mathrm{B}$ chain.

Peptide displacement studies. Based on the knowledge (see results) that the binding of phagotopes to IAS involved concensus sequences incorporated within the last 18 residues of the insulin B chain, notably B20-23, we conducted serial dilution displacement studies on IAS using synthetic peptides comprising the terminal six (B25-30), nine (B22-30), 12 (B19-30), 15 (B16-30) and 18 (B13-30) residues. The results were expressed as the molarity of each needed to achieve $50 \%$ displacement of the radiollabelled human insulin. 
Table 1. Binding in the capture ELISA of phagotopes selected by the affinity-purified sera of two patients: IAS and IDD with their corresponding peptide sequences. Assay signals are expressed as standard deviation scores (SDS) of the mean of normal sera

\begin{tabular}{lcl}
\hline Phagotopes & SDS & Sequence \\
\hline IAS test serum & & \\
IAS-1 & 0.4 & GKGAGDL \\
IAS-2 & 0.8 & VQKDVLL \\
IAS-3 & 1.6 & R*QLGPD \\
IAS-4 & 2.2 & *GSDR*D \\
IAS-5 & 3.2 & VISRSRV \\
IAS-6 & 2.2 & AALGSAI \\
IAS-7 & 3.5 & GRTSRTV \\
IAS-8 & 2.9 & IPDSRLK \\
IAS-9 & 11.8 & KRSRLDV \\
IAS-10 & 2.7 & *IGRGSR \\
IDD serum & & \\
IDD-1 & & LDYNE*P \\
IDD-2 & 1.1 & *IGLGRG \\
IDD-3 & 3.1 & KLGRG*V \\
IDD-4 & 2.6 & DG-6QPSFP \\
IDD-5 & 0.9 & LKGSG*V \\
IDD-6 & 4.2 & RLGKRDS \\
IDD-7 & 2.5 & YLGRGDK \\
IDD-8 & 3.9 & RQLGRGS \\
IDD-9 & 6.8 & STVQDCV \\
IDD-10 & 0.6 & LGRGGSK \\
\hline & 8.4 & \\
\hline & &
\end{tabular}

\section{Results}

Insulin binding and insulin species displacement studies with IAS and IDD. IAS bound $19.5 \%$ of the radiolabelled insulin (mean of two replicate assays). The molarities of human, porcine and bovine insulin needed to displace $50 \%$ of the binding were $1.8 \pm 0.3 \times 10^{-10} \mathrm{~mol} / \mathrm{l}$, $3.5 \pm 1.1 \times 10^{-9} \mathrm{~mol} / \mathrm{l}$ and $3.8 \pm 1.7 \times 10^{-9} \mathrm{~mol} / 1 \mathrm{respec}-$ tively.

IDD bound $13.3 \%$ of the radiolabelled insulin (mean of two replicate assays). The molarities of human, porcine and bovine insulin needed to displace $50 \%$ of the binding were $3.3 \pm 0.5 \times 10^{-9} \mathrm{~mol} / \mathrm{l}, 3.7 \pm 0.8 \times 10^{-9} \mathrm{~mol} / 1$ and $2.9 \pm 1.1 \times 10^{-9} \mathrm{~mol} / \mathrm{l}$ respectively.

Immunospecificity of reaction between phage and IAS/ $I D D$. Of ten phagotopes isolated by biopanning with purified IAS, seven bound the IAS with a signal exceeding 2 SDS in the capture ELISA: IAS-4, IAS-5, IAS-6, IAS-7, IAS-8, IAS-9 and IAS-10 (Table 1). The binding of each could be displaced as much with porcine and bovine as with human insulin (data not shown), suggesting that the phagotopes selected by IAS were bound by the IA rather than the IAA it contained.

Similarly, seven IDD phagotopes bound IDD with a signal exceeding 2SDS: IDD-2, IDD-3, IDD-5, IDD-6, IDD-7, IDD-8 and IDD-10 (Table 1), and in each the binding could be displaced equally with cold human, porcine and bovine insulin (data not shown).
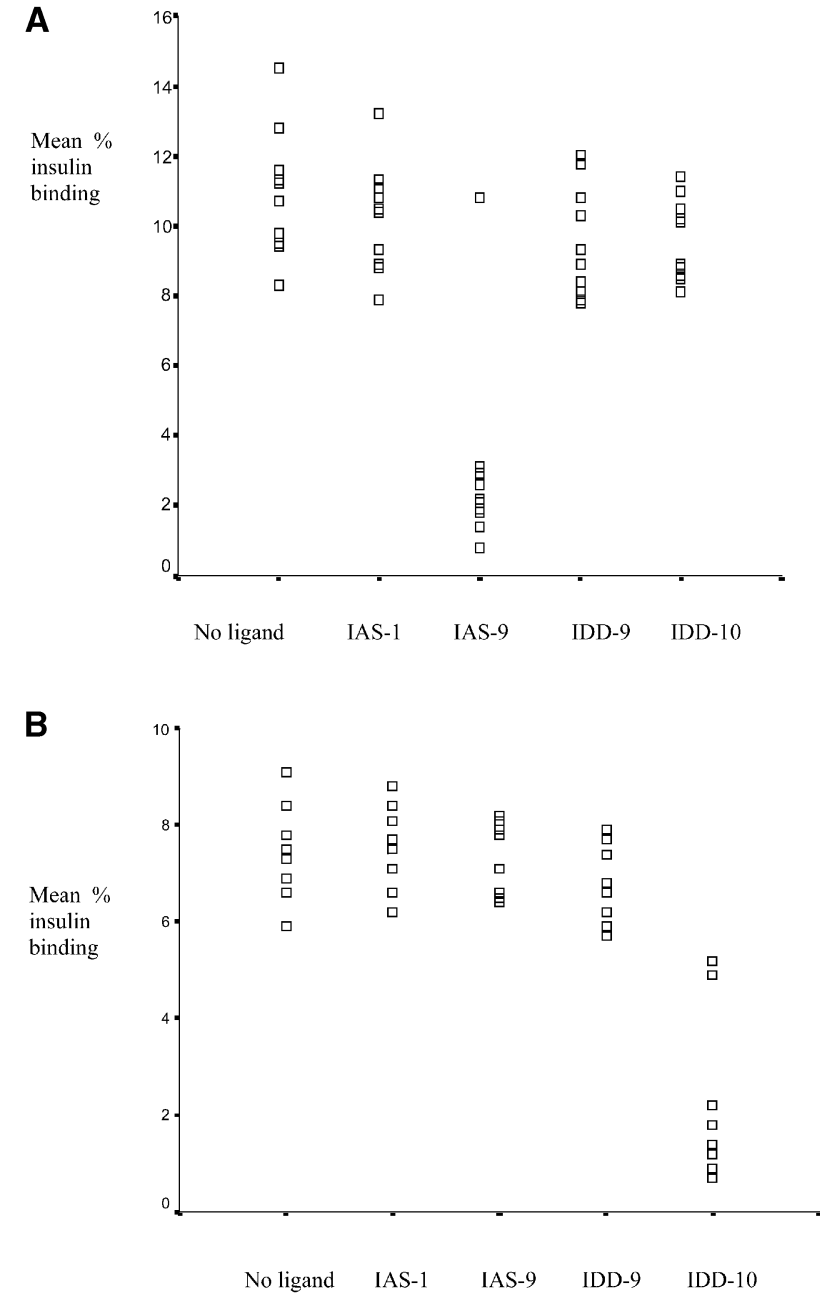

Fig. 2A, B. The ability of phagotopes (dilution 1:100) selected by IAS (IAS-1, IAS-9) and IDD (IDD-9 and IDD-10) to displace the binding of human insulin by the sera of ten $\mathrm{IA}^{+}$ patients with insulin-treated Type 2 diabetes (A), and eight diabetes-associated $\mathrm{IAA}^{+}$sera $(\mathbf{B})$

Discrimination of IA from IAA by phagotopes. The phagotopes selected for their reactivity in the capture ELISA were used for displacement studies in the RBA. Of the ten phagotopes selected by IAS, IAS-9 competed most strongly for insulin binding to IAS, and IAS-1 the least (Fig. 1A). Similarly, IDD-10 competed the most strongly for insulin binding to IDD and IDD-9 the least (Fig. 1B). The amount of phage particles at the dilution of $1: 1$ in these four phagotopes-IAS-1,IAS-9, IDD-9 and IDD-10 was $4.8 \times 10^{5}$, $3.6 \times 10^{5}, 5.4 \times 10^{5}$ and $3.9 \times 10^{5}$ respectively.

IAS-1 and IAS-9 were used in studies attempting to displace the binding of labelled insulin to IA or IAA in IAS and sera of the eight patients with newly diagnosed Type 1 diabetes and ten patients with insulintreated Type 2 diabetes. At a dilution of 1:100, IAS-9 was able to displace insulin binding in sera from nine out of ten patients with insulin-treated Type 2 diabetes, whereas IAS-1 was unable to displace insulin binding in any of them (Fig. 2A). On the other hand, 


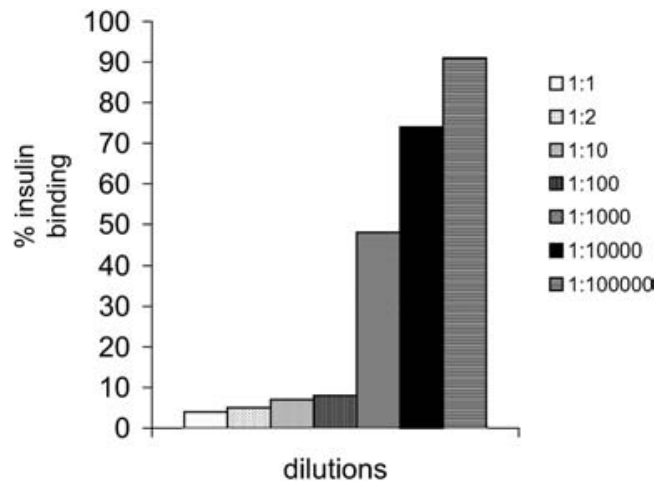

Fig. 3. Displacement of insulin binding in an individual with insulin antibody in a radiobinding assay using phagotopes IAS-9 at various dilutions

neither IAS-9 nor IAS-1 was able to displace insulin binding in any of the sera from patients with Type 1 diabetes. We showed that at various dilutions of IAS-9, displacement of insulin binding using a RBA in an individual with IA, is increased with increasing concentrations of the phagotope (Fig. 3).

Neither IDD-10 nor IDD-9, was able to displace insulin binding in any of the sera from the ten patients with insulin-treated Type 2 diabetes. IDD-10 did, however, displace insulin binding in the sera from all eight newly diagnosed Type 1 diabetes patients (Fig. 2B) while IDD-9 was unable to.

Thus, between them, just two phagotopes could distinguish between three insulin-binding idiotypes, only one of them predictive of diabetes. Phagotope IAS-9 was able to positively discriminate IA from IAA and IDD-10 to positively discriminate diabetesrelated IAA from IA. None of the phagotopes was able to displace the binding of IAS to human insulin, but this particular IAA idiotype could be discriminated by the failure of porcine insulin to displace it.

DNA sequencing of phagotopes. We examined the sequences of the seven IAS-reactive phagotopes selected by IAS for consensus. The sequence SR was shared by five of them (Table 1), RSR by two (IAS-5 and IAS-9), SRL by two (IAS-9 and IAS-8), GS by three (IAS-4, IAS-6 and IAS-10) and GR by two (IAS-7 and IAS-10). The peptide KRSRLDV (IAS-9) showed the strongest reaction with the IA-containing serum IAS (11.75 SDS). None of the three phagotopes nonreactive with IAS shared peptide sequences with the IAS-reactive phagotopes (Table 1).

The sequence GS was also represented in five of the seven IDD-reactive phagotopes selected by IDD. The sequence LGRG was shared by five of the IDD-reactive phagotopes, among which IDD-10 (LGRGGS) had the strongest reaction with serum IDD (8.4 SDS). There was no sharing of sequences between the IDD-reactive and non-reactive phagotopes, except for peptide GS incorporated in IDD-4.
Table 2. Epitope mapping of the insulin A and B chain according to consensus sequences of phagotopes reactive with serum IAS (2A) and serum IDD (2B)

\begin{tabular}{ll}
\hline $\begin{array}{l}\text { Consensus } \\
\text { sequence }\end{array}$ & $\begin{array}{l}\text { Candidate epitope domains on A or B chain } \\
\text { of insulin }\end{array}$
\end{tabular}

\begin{tabular}{ll}
\hline 2A. IAS & \\
GR & B 20-B 22; B 22-B 23 \\
GS & A1-A9; A 1-A12; B 8-B9; B9-B20; B 9-B 23 \\
SR & B 9-B 22 \\
SRL & B 6-B 9-B 22; B 9-B 11-B 22; \\
& B 9-B 17-B 22
\end{tabular}

2B. IDD

LGRG B6-B20-B22-B23; B11-B20-B22-B23; B17-B20-B22-B23

We next compared the consensus sequences of phagotopes which bound to IAS (GR, GS, SR) and to IDD(LGRG) with those of the insulin A and B chain with a view to identifying possible points of contact between epitope and idiotope (Table 2).

Peptide displacement studies. The molarities of whole human insulin, the peptide B18-30 and the peptide B23-30 required to displace $50 \%$ of the radiolabelled human insulin were $3.8 \times 10^{-4}, 4.3 \times 10^{-2}$ and $1.1 \times 10^{2} \mu \mathrm{mol} / \mathrm{l}$ respectively.

\section{Discussion}

We have used two insulin-purified IgG preparations containing respectively a mix of human insulin specific IAA/IA and non-human insulin specific, diabetesrelated, IAA to screen a phage-display library of random heptapeptides expressed in the pIII coat protein of bacteriophage. The phagotopes selected by these antibody preparations were sequenced and tested for their ability to displace insulin binding from the source serum. The phagotope IAS-9 (sequence KRSRLDV) showed the strongest reaction with the insulin-purified IgG extract of IAS, identified IA (insulin-treated Type 2 subjects) but not diabetes-related IAA (insulin naive Type 1 diabetic subjects). In contrast, the phagotope IDD-10 identified diabetes-related IAA in the sera of the newly diagnosed diabetic subjects, but neither IA in the sera of insulin-treated subjects, nor the human-insulin specific, diabetes-unrelated IAA in IAS. Thus, the two reagents IAS-9 and IDD-10 between them were able to discriminate, both positively and negatively, between diabetes-associated and non-diabetes associated insulin antibodies. We believe this is the first demonstration of reagents capable of doing so.

There have been previous attempts to characterize antigenic epitopes relevant to Type 1 diabetes [21]. Phage display technology has helped to improve the 
prognostic value of IA-2 antibodies, another serological marker in Type 1 diabetes [22], making it possible to distinguish diabetes-related from diabetes-unrelated antibodies. The present report suggests that the same can be achieved with insulin antibodies.

It is possible that some of the sequences derived from the phage library will represent phagotopes for antibodies other than IAA or IA. This is inevitable in the first instance, given the heterogeneity of the normal immune response, and it remains the major limitation of applying phage display to individual sera. We have tried to minimise the risk of misinterpretation in two ways, firstly by affinity purification of the screening sera with human insulin and secondly by showing that the binding of phagotope to antibody could be displaced in a dose-dependent manner by purified insulin. Displacement by insulin as a ligand is strong evidence of specificity. It is worth noting that we tested only two sets of sera with a specific HLA haplotype. It will be interesting to establish if there is a difference in dominant phagotopes sequenced from sera of different HLA haplotypes. With reference specifically to IAA and IAS, HLA associations have been shown to strongly associated, especially in the Japanese population. For instance, glutamate at position 74 in the HLA-DR4 beta 1 chain was presumed to be associated with polyclonal IAA production, whereas alanine at the same position might be important in the production of monoclonal IAA [22].

Deducing which of the epitopes on the phagotope were recognised by the screening antibodies is facilitated by the knowledge of the structure of insulin and by affinity purification of the sera. From the IAS patient, consensus region GR could represent positions 20 and 22 of the insulin B chain, or positions 22 and 23. GR is unlikely to represent a possible epitope on the A chain of the insulin molecule, as arginine is not represented on the human insulin A chain. The sequence GS, an epitope reactive with both both IAS and IDD, could represent positions 9 and 13 or 9 and 21 on the insulin B chain and position 1 and 9 or 1 and 12 on the insulin A chain. The most commonly shared sequence, SR, could represent position 9 and 22 on the B chain. In contrast, SR was unlikely to represent an epitope on the A chain as again, arginine is not found on the insulin A chain. Threonine, position 30 on the human insulin $B$ chain and the residue uniquely human among the three insulin variants used in the initial displacement studies on IAS and IDD, was represented in none of the phagotope sequences. The sequence LGRG, shared by five of the IDD-reactive phagotopes, could represent position 6/11/17(Leucine), 20(Glycine), 22(Arginine) or 23(Glycine) of the insulin B chain. LGRG is unlikely to represent a linear epitope of the insulin A chain as arginine is not present on the A chain.

Glutamate 21 and arginine 22 are located at a type 1 beta turn formed between residues glycine B20 and glycine B23. This is the so-called beta-sheet hinge, the flexibility of which is important in the binding of insulin to its receptor and thus its biological activity in vivo [24]. Conformational flexibility permits the terminal residues of the B chain, which are not involved in receptor binding, to swing away from the hydrophobic region located underneath [25], allowing underlying residues essential for receptor binding to become more accessible. The exposure of this aliphatic area is also important in the formation of insulin dimers, a process that alters the solubility and half-life of insulin in vivo [26]. Antibody binding to the terminal $\mathrm{B}$ chain might therefore be expected to block the biological activity of insulin consistent with antibodymediated insulin resistance seen as a characteristic of insulin autoimmune syndrome. Glycine and arginine were shared by the phagotope sequences reactive with both IAA and IA, suggesting that beta-sheet hinge of the insulin molecule could be universally immunogenic. Furthermore, the displacement data using peptides of different length from the amino-terminal of the B chain suggest that the deletion of arginine, in particular, has substantial impact on the binding of insulin in the IAS serum. IAS showed far greater affinity (that is it was able to displace human insulin at far lower concentration) for the synthetic oligopeptide B18-30 than for B23-30, and greater again for the intact insulin molecule.

The phagotopes selected by the diabetes-related IAA from serum IDD showed a consensus sequence (LGRG) that was strikingly different from those selected by the mix of human insulin specific IAA and IA in serum IAS, supporting the precept that insulin antibodies found in different clinical conditions are idiotypically distinct and recognise different epitopes on the insulin molecule. On the other hand, the idiotypes of antibodies from people with the same clinical condition seem to be remarkably uniform. A previous study showed that the lymphocytes committed to the production of IgG autoantibodies to insulin are common in the beta-cell repertoire at the onset of diabetes [27].

All reactive phagotopes sequenced in this study were cross-reactive with human, porcine and bovine insulin, as shown by their displacement with the three variants in the capture ELISA. This plurality of binding suggests that biopanning of IAS preferentially selected its IA moiety, rather than the human insulin specific (B-30 threonine-dependent) IAA present in this serum and characterised previously in detail [15]. This conclusion is consistent with the observation that phagotope IAS-9 competed strongly with IA for insulin binding in patients with insulin treated Type 2 diabetes. Phage display is an affinity dependent technique, and failure of the serum to select a human insulin B-30 mimotope, despite serological evidence that a human insulin-specific antibody was present, suggests that B30-dependent reactivity may be of relatively 
low affinity, albeit high capacity in IAS. Others found from Scatchard analysis that the affinity of IA from a patient with insulin treated diabetes was much higher that of IAA found in the insulin autoimmune syndrome [28]. In conclusion, this study provides early evidence (proof of principle) that disease-specific insulin binding idiotopes can be detected by phage technology. It points the way to studies using site-directed mutagenesis with which to resolve yet further the epitopes involved in IA/IAA binding, and the development of reagents which could distinguish diabetespredictive IAA.

Acknowledgements. These studies were carried out as part of the EC-funded DiabMarker program (CT 983363) in collaboration with T. Mandrup-Poulsen (Copenhagen), H. Kolb (Dusseldorf), B. Roep (Leiden), M.Knip (Helsinki) and E. Bonifacio (Milan). We are grateful to Dr.H. Grayson for his assistance with DNA purification, to Dr. M. Rowley for providing the details for the capture ELISA protocol and to Prof. M. Knip for providing the IDD sera. This study was supported by the Sir Halley Stuart Trust and the Northcott Devon Medical Foundation.

\section{References}

1. Report of the Expert Committee on the Diagnosis and Classification of Diabetes Mellitus (1993) Diabetes Care 20:1183-1197

2. Bonifacio E, Christie MR (1997) Islet cell antigens in the prediction and prevention of insulin-dependent diabetes mellitus. Ann Med 29:405-412

3. Palmer JP, Asplin CM, Clemons P et al. (1983) Insulin antibodies in insulin-dependent diabetics before insulin treatment. Science 222:1337-1339

4. Ziegler AG, Hummel M, Schenker M, Bonifacio E (1999) Autoantibody appearance and risk for development of childhood diabetes in offspring of parents with type 1 diabetes: the 2-year analysis of the German BABYDIAB Study. Diabetes 48:460-468

5. Wilkin TJ (1991) Autoantibodies as mechanisms, markers, and mediators of B-cell disease. Diabetes Metab Rev $7: 105-120$

6. Naserke HE, Dozio N, Ziegler AG, Bonifacio E (1998) Comparison of a novel micro-assay for insulin autoantibodies with the conventional radiobinding assay. Diabetologia 41:681-683

7. Yu L, Robles DT, Abiru N et al. (2000) Early expression of antiinsulin autoantibodies of humans and the NOD mouse: evidence for early determination of subsequent diabetes. Proc Natl Acad Sci USA 97:1701-1706

8. Williams AJ, Bingley PJ, Bonifacio E, Palmer JP, Gale EA (1997) A novel micro-assay for insulin autoantibodies. J Autoimmun 10:473-478

9. Diaz JL, Wilkin T (1987) Differences in epitope restriction of autoantibodies to native human insulin (IAA) and antibodies to heterologous insulin (IA). Diabetes 36:6672

10. Nell LJ, Hulbert C, Thomas JW (1989) Human insulin autoantibody fine specificity and $\mathrm{H}$ and $\mathrm{L}$ chain use. J Immunol 142:3063-3069
11. Schroer JA, Bender T, Feldmann RJ, Kim KJ (1983) Mapping epitopes on the insulin molecule using monoclonal antibodies. Eur J Immunol 13:693-700

12. Castano L, Ziegler AG, Ziegler R, Shoelson S, Eisenbarth GS (1993) Characterization of insulin autoantibodies in relatives of patients with type I diabetes. Diabetes 42:12021209

13. Thomas JW (2001) Antigen-specific responses in autoimmunity and tolerance. Immunol Res 23:235-244

14. Hirata Y, Nishimura H, Tominaga M, Ishizu H, Arimichi M (1972) Spontaneous hypoglycemia with insulin-autoimmunity. Nippon Naika Gakkai Zasshi 61:1296-1304

15. Sklenar I, Wilkin TJ, Diaz JL, Erb P, Keller U (1987) Spontaneous hypoglycemia associated with autoimmunity specific to human insulin. Diabetes Care 10:152-159

16. Scott JK, Smith GP (1990) Searching for peptide ligands with an epitope library. Science 249:386-390

17. Osman AA, Uhlig H, Thamm B, Schneider-Mergener J, Mothes T (1998) Use of the phage display technique for detection of epitopes recognized by polyclonal rabbit gliadin antibodies. FEBS Lett 433:103-107

18. Folgori A, Tafi R, Meola A et al. (1994) A general strategy to identify mimotopes of pathological antigens using only random peptide libraries and human sera. EMBO J 13:22362243

19. Geysen HM, Rodda SJ, Mason TJ (1986) The delineation of peptides able to mimic assembled epitopes. Ciba Found Symp 119:130-49

20. Rowley MJ, Scealy M, Whisstock JC et al. 2000 Prediction of the immunodominant epitope of the pyruvate dehydrogenase complex E2 in primary biliary cirrhosis using phage display. J Immunol 164:3413-3419

21. Mennuni C, Santini C, Lazzaro D et al. 1997 Identification of a novel type 1 diabetes-specific epitope by screening phage libraries with sera from pre-diabetic patients. J Mol Biol 268:599-606

22. Miao D, Yu L, Tiberti C et al. (2002) ICA512(IA-2) Epitope specific assays distinguish transient from diabetes associated autoantibodies. J Autoimmun 18:191-196

23. Uchigata Y, Tokunaga K, Nepom G et al. (1995) Differential immunogenetic determinants of polyclonal insulin autoimmune syndrome and monoclonal insulin autoimmune syndrome. Diabetes 44:1227-1232

24. Baker EN, Blundell TL, Cutfield JF et al. (1988) The structure of 2Zn pig insulin crystals at $1.5 \mathrm{~A}$ resolution. Philos Trans R Soc Lond B Biol Sci 319:369-456

25. Kurapkat G, De Wolf E, Grotzinger J, Wollmer A (1997) Inactive conformation of an insulin despite its wild-type sequence. Protein Sci 6:580-587

26. Hua QX, Shoelson SE, Weiss MA (1992) Nonlocal structural perturbations in a mutant human insulin: sequential resonance assignment and 13C-isotope-aided 2D-NMR studies of $[\mathrm{PheB} 24 \rightarrow$ Gly]insulin with implications for receptor recognition. Biochemistry 31:11940-11951

27. Casali P, Nakamura M, Ginsberg-Fellner F, Notkins AL (1990) Frequency of B cells committed to the production of antibodies to insulin in newly diagnosed patients with insulin-dependent diabetes mellitus and generation of high affinity human monoclonal IgG to insulin. J Immunol 144:3741-3747

28. Eguchi Y, Uchigata Y, Yao K et al. (1994) Longitudinal changes of serum insulin concentration and insulin antibody features in persistent insulin autoimmune syndrome (Hirata's disease). Autoimmunity 19:279-284 